\title{
MicroRNA-10a silencing reverses cisplatin resistance in the A549/cisplatin human lung cancer cell line via the transforming growth factor- $\beta /$ Smad2/STAT3/STAT5 pathway
}

\author{
WEI SUN ${ }^{1 *}$, YIPING MA ${ }^{2 *}$, PENG $\mathrm{CHEN}^{3}$ and DONG WANG ${ }^{4}$ \\ ${ }^{1}$ Department of Thoracic Surgery, Tianjin Chest Hospital, Tianjin 300051; ${ }^{2}$ Department of Pharmacy, \\ Tianjin Huanhu Hospital, Tianjin 300060; Departments of ${ }^{3}$ Thoracic Medical Oncology and ${ }^{4}$ Pharmacy, \\ Tianjin Medical University Cancer Institute and Hospital, Key Laboratory of Cancer Prevention and Therapy, \\ Tianjin 300060, P.R. China
}

Received January 12,2014; Accepted November 7, 2014

DOI: $10.3892 / \mathrm{mmr} .2015 .3181$

\begin{abstract}
Lung cancer is one of the primary causes of mortality worldwide and drug resistance is the key contributing factor which results in the failure of lung cancer chemotherapy. Previous studies have shown that microRNA (miR)-10a was involved in the reversal of cisplatin (DDP) resistance in numerous types of tumors; however, the underlying mechanism of action of this remains to be fully elucidated. In the present study, miR-10a silencing in human DDP-resistant lung cancer A549/DDP cells was demonstrated to improve DDP sensitivity, apoptosis, intracellular rhodamine-123 content as well as the expression and activity of caspase-3/8. In addition, miR-10a suppressed the cellular expression of P-glycoprotein, multi-drug resistance protein (MDR) 1, MDR-associated protein 1, RhoE, B cell lymphoma-2 and survivin in A549/DDP cells. Furthermore, miR-10a silencing inhibited the secretion of transforming growth factor (TGF)- $\beta$, phosphorylation of Sma- and Mad-related protein (Smad)2, signal transducer and activator of transcription (STAT) 3 and STAT5, the transcriptional activity of hypoxia-inducible factor and eukaryotic translation initiation factor $4 \mathrm{E}$ in human lung cancer A549/DDP cell line. These results therefore indicated that miR-10a may be a potential target for improving the effectiveness of lung cancer chemotherapy via regulation of the TGF- $\beta /$ Smad2/STAT3/STAT5 pathway.
\end{abstract}

Correspondence to: Mr. Dong Wang, Department of Pharmacy, Tianjin Medical University Cancer Institute and Hospital, Key Laboratory of Cancer Prevention and Therapy, Huanhu West Street, Tianjin 300060, P.R. China

E-mail: wangdong_tmu@126.com

*Contributed equally

Key words: microRNA-10a, human lung cancer, chemotherapy resistance

\section{Introduction}

MicroRNAs (miRNAs) are a type of non-coding small-fragment RNA with 21 basic groups, which are transcribed by cells in order to alter gene expression, predominantly through post-transcriptional regulation (1). With the increase of studies focusing on this field of research, miRNAs have been confirmed to be involved in a variety of physiological and pathological activities, including cell proliferation, apoptosis, cell cycle regulation, tumor formation and inflammation (2). One previous study demonstrated that miRNA, including miR205, was closely associated with tumor development; in addition, the involvement of miRNAs in tumor formation can be classified into two categories according to their different effects: Cancer-promoting and -suppressing miRNAs $(3,4)$.

miRNA (miR)-10a novel microRNA, which was found to be closely associated with tumor growth, survival, invasion and metastasis. A recent study demonstrated that miR-10a promoted tumor growth, metastasis and invasion in cervical cancer, indicating that miR-10a may be an important cancer-promoting miRNA (5).

Although antitumor drugs, including cisplatin, have been highly effective and widely applied in the treatment of lung cancer, drug-resistance has become an increasingly prominent issue, as it is a key obstacle in the effective treatment of cancer patients. Therefore, multidrugresistance (MDR) reversal is significant in the development of tumor therapies. The major mechanisms underlying tumor MDR, include incerased drug excretion, decreased drug intracellular concentration and increased expression of antiapoptotic factors in tumor cells (6). Furthermore, the role of miR-10a in tumor cell apoptosis and drug resistance remains to be elucidated. The present study aimed to explore the role and mechanisms of miR-10a in drug-resistance reversal of non-small cell lung cancer cells.

\section{Materials and methods}

Cell lines and cell culture. Human lung cancer A549 and human cisplatin (DDP)-resistant lung cancer A549/DDP cells were purchased from the Cell Bank of Chinese Academy of 
Science (Shanghai, China) and cultured at $37^{\circ} \mathrm{C}$ with $5 \% \mathrm{CO}_{2}$ in Dulbecco's modified Eagle's medium (DMEM; GibcoBRL, Invitrogen Life Technologies, Carlsbad, CA, USA) with $10 \%$ fetal bovine serum (FBS; GibcoBRL). miR-10a antisense oligonucleotides (miR-10a inhibitor) and the miRNA control were purchased from Shanghai GenePharma Co., Ltd (Shanghai, China) and transfected into cells using a Lipofectamine $^{\mathrm{TM}} 2000$ assay (Invitrogen Life Technologies, Carlsbad, CA, USA). The groups were as follows: Parent group, A549 cells; control group, A549/DDP cells without treatment; negative control (NC) group, A549/DDP cells transfected with miR-10a (ASO-miR-NC); and miR-10a inhibitor group, A549/DDP cells transfected with an miR-10a inhibitor (ASO-miR-10a).

miRNA was extracted from cells using a TaqMan ${ }^{\circledR}$ miRNA isolation kit (Invitrogen Life Technologies) and the expression of miR-10a was measured using a TaqMan ${ }^{\circledR}$ Universal polymerase chain reaction (PCR) Master Mix assay (Invitrogen Life Technologies). U6 was used as the internal reference gene.

MTS assay. Cells $\left(0.5 \mathrm{ml} ; 1 \times 10^{4} / \mathrm{ml}\right)$ were seeded onto a 96-well microplate and incubated overnight at $37^{\circ} \mathrm{C}$ with $5 \%$ $\mathrm{CO}_{2}$. Various concentrations of DDP $(0,5,10,20,50$ and $100 \mu \mathrm{M}$; SigmaAldrich, St. Louis, MO, USA) were added and cultured for $72 \mathrm{~h}$. Fresh culture medium with $20 \mu \mathrm{l}$ MTS (SigmaAldrich) was then added and cells were incubated for $4 \mathrm{~h}$. Absorbance values were determined using a microplate reader (Model 680; BioRad Laboratories, Inc., Hercules, CA, USA) at $490 \mathrm{~nm}$ in order to calculate the rate of cell proliferation inhibition.

Flow cytometric analysis. Flow cytometry was used to analyze the apoptotic rate of cells. Cells $\left(0.3 \mathrm{ml} ; 1 \times 10^{6} / \mathrm{ml}\right)$ were seeded onto a six-well microplate for $24 \mathrm{~h}$ and exposed to $10 \mu \mathrm{M}$ DDP for a further $24 \mathrm{~h}$. Cells were then centrifuged at $1000 \mathrm{x} \mathrm{g}$ for $5 \mathrm{~min}$ at room temperature, collected and stained using a fluorescein isothiocyanate (FITC)/propidium iodide (PI) apoptosis detection kit (BD Biosciences, Franklin Lakes, NJ, USA) for $15 \mathrm{~min}$ in the dark. Fluorescence intensity was the measured using a flow cytometer (FACSCalibur; BD Biosciences).

For detection of intracellular rhodamine (Rh)-123 content and P-glycoprotein (P-gp) expression, cells $\left(0.3 \mathrm{ml} ; 1 \times 10^{6} / \mathrm{ml}\right)$ were seeded onto a six-well microplate for $24 \mathrm{~h}$ and then centrifuged at $300 \mathrm{xg}$ for $10 \mathrm{~min}$, collected and incubated with rabbit antihuman polyclonal Rh-123- or P-pg-phycoerythrin antibodies (Beyotime Institute of Biotechnology, Shanghai, China) for $30 \mathrm{~min}$ in the dark. Fluorescence intensity was then measured using a flow cytometer.

Caspase-3/8 activity was measured using an active caspase-3/8 apoptosis kit (Xiamen Lulong Biotech Development Co., Ltd, Xiamen, China) according to the manufacturer's instructions In brief, cells $\left(0.3 \mathrm{ml} ; 1 \times 10^{6} / \mathrm{ml}\right)$ were seeded onto a six-well microplate for $24 \mathrm{~h}$. Cells were then permeabilized, fixed and incubated with active caspase-3-FITC or active caspase-8-FITC antibodies for $30 \mathrm{~min}$ in the dark. Fluorescence intensity was then measured using a flow cytometer.

Western blot analysis. Cells $\left(0.3 \mathrm{ml} ; 1 \times 10^{6} / \mathrm{ml}\right)$ were seeded onto a six-well microplate for $24 \mathrm{~h}$ and lysed using a lysate buffer (Beyotime Institute of Biotechnology);100 $\mu \mathrm{g}$ total cell lysate was the separated using 12\% SDS-PAGE (Beyotime Institute of Biotechnology). Proteins were then transferred onto a polyvinylidene fluoride membrane (Millipore, Billerica, MA, USA) and blocked using 5\% non-fat milk for $1 \mathrm{~h}$ at $4^{\circ} \mathrm{C}$. The membrane was incubated with rabbit antihuman polyclonal primary antibodies (MDR1, 1:1,000; MDR-associated protein (MRP)1, 1:1,000; RhoE, 1:1,000; B cell lymphoma 2 (Bcl-2), 1:1,000; Survivin, 1:1,000; caspase-3, 1:1,000; caspase-8, 1:1,000; p53, 1:1,000; phosphorylatedsignal transducer and activator of transcription (p-STAT) 3 , 1:1,000; and p-STAT5, 1:1,000; Cell Signaling Technologies, Danvers, MA, USA) and $\beta$-actin (1:5,000; SigmaAldrich) at $4^{\circ} \mathrm{C}$ overnight. The membranes were then washed three times in 5\% phosphate-buffered saline with Tween-20 (Beyotime Institute of Biotechnology) and incubated for $1 \mathrm{~h}$ with horseradish peroxidase-conjugated anti-rabbit secondary antibodies (1:5,000; Cell Signaling Technologies) at room temperature. Western blots were visualized using an Enhanced Chemiluminescence Western Blotting kit (GE Healthcare, Little Chalfont, UK). $\beta$-actin was as the internal control.

Quantitative PCR $(q P C R)$. Cells $\left(0.3 \mathrm{ml} ; 1 \times 10^{6} / \mathrm{ml}\right)$ were seeded onto a six-well microplate for $24 \mathrm{~h}$ and total RNA was extracted using a TRIzol ${ }^{\circledR}$ assay (Invitrogen Life Technologies, Carlsbad, CA, USA). qPCR was performed using a Mastercycler Gradient (nexus G2; Eppendorf, Hamburg, Germany). GAPDH was amplified as the internal control. The primer sequences were as follows: MDR1 forward, 5'-CACCTTAAAGGGCCA CAG-3' and reverse, 5'-TGCCGACCGTACAAGAGT-3'; MRP1 forward, 5'-AGGTCTGCCCAGCAGACGATCCA-3' and reverse, 5'-GGACAAGCACTGAAAGATAAGAAAGA-3'; RhoE forward, 5'-ACACATATGAAGGAGAGAA -3' and reverse, 5'-TAAGGCGGCCGCAACATGA-3'; Bcl-2 forward, 5'-GGTGAACTGGGGGAGGATTGT-3' and reverse, 5'-CTT CAGAGACAGCCAGGAGAA-3'; Survivin forward, 5'-CTC TACATTCAAGAACTGGCC-3' and reverse, 5'-TTGGCTCTT TCTCTGTCCAG-3'; GAPDH forward, 5'-TGCACCACCAAC TGCTTAGC-3' and reverse, 5'-GGCATGGACTGTGGTCAT GAG-3'. Following 5 min of denaturation, 30 cycles of amplification were performed, each cycle consisted of: $55 \mathrm{sec}$ at $95^{\circ} \mathrm{C}$, $40 \mathrm{sec}$ at $55^{\circ} \mathrm{C}$ and $65 \mathrm{sec}$ at $72^{\circ} \mathrm{C}$; the reaction was activated at $72^{\circ} \mathrm{C}$ for $5 \mathrm{~min}$ and terminated at $4^{\circ} \mathrm{C}$.

Reporter gene assay. Cells $\left(0.3 \mathrm{ml} ; 1 \times 10^{6} / \mathrm{ml}\right)$ were seeded onto a six-well microplate for $24 \mathrm{~h}$. Luciferase reporter plasmids (Beyotime Institute of Biotechnology) for hypoxia-inducible factor (HIF) and eukaryotic translation initiation factor 4E (eIF4E) as well as Renilla luciferase plasmids were transfected into cells using Lipofectamine ${ }^{\mathrm{TM}} 2000$ and incubated for $24 \mathrm{~h}$. Cells were then centrifugated, collected and the fluorescence intensity of fluorescein was detected using a dual luciferase assay kit. Renilla luciferase was used as the internal control.

ELISA assay. Transforming growth factor (TGF)- $\beta$ detection was performed using an ELISA kit (R\&D Systems, Minneapolis, MN, USA) according to the manufacturer's instructions. In brief, cells $\left(0.3 \mathrm{ml} ; 1 \times 10^{6} / \mathrm{ml}\right)$ were seeded onto a six-well microplate for $24 \mathrm{~h}$, the serum-free medium was replaced for and cells were 


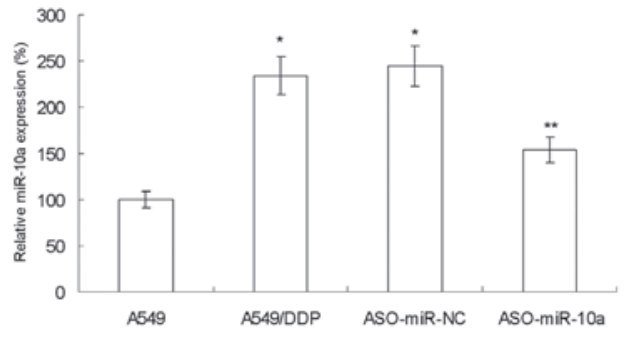

Figure 1. miR-10a expression is increased in DDP-resistant lung cancer A549/DDP cell lines. Quantitative polymerase chain reaction showed an increase in miR-10a expression in DDP-resistant A549/DDP cells compared with the A549 cell line. Following transfection with an miR-10a inhibitor (AOS-miR-10a), miR-10a expression was decreased in A549/DDP cells. Values are presented as the mean \pm standard deviation $(n=10) ;{ }^{*} \mathrm{P}<0.05$ vs. the A549 group and ${ }^{* *} \mathrm{P}<0.05$ vs. the A549/DDP group. DDP, cisplatin A549 group, control; A549/DDP group, DDP-resistant A549 cells without treatment; ASO-miR-NC group, negative control group of A549/DDP cells transfected with miR-10a; ASO-miR-10a group, A549/DDP cells transfected with an miR-10a inhibitor; miR, microRNA.

incubated for a further $24 \mathrm{~h}$. Absorbance was then determined using a microplate reader at $490 \mathrm{~nm}$.

Statistical analysis. A one-way analysis of variance was used to determine differences between groups. Data analyses were performed using SPSS 11.0 statistical software (SPSS Inc., Chicago, IL, USA). P<0.05 was considered to indicate a statistically significant difference between values.

\section{Results}

Increased expression of miR-10a in lung cancer DDP-resistant A549/DDP cells. In order to study the drug-resistance reversal effect of miR-10a, the expression of miR-10a was examined in A549 and DDP-resistant A549/DDP cells. As shown in Fig. 1, the expression of miR-10a in A549/DDP cells was significantly increased compared with that of A549 cells, indicating that miR-10a may be closely associated with DDP-resistance.

MiR-10a silencing increases DDP sensitivity in A549/DDP cells. An miR-10a antisense oligonucleotide (ASO-miR-10a) was used to silence the expression of miR-10a in A549/DDP cells. Cell viability was the assess in miR-10a cells treated with various concentrations of DDP (Fig. 2A). The results showed that following silencing miR-10a, the sensitivity of A549/DDP cells to DDP was significantly enhanced. In addition, the effect of miR-10a silencing on DDP-induced apoptosis was investigated (Fig. 2B). The results showed that miR-10a silencing increased the apoptotic rate of A549/DDP cells. This therefore suggested that miR-10a had an important role in DDP-resistance inA549/DDP cells.

Bcl-2 and survivin are two important families involved in the regulation of cell apoptosis $(7,8)$; in addition, $\mathrm{RhoE}$ also has an important role in tumor drug resistance (9). In the present study, the effect of miR-10a silencing on the protein and messenger (m) RNA expression of Bcl-2, survivin and RhoE was investigated in DDP-resistant A549/DDP cells (Fig. 2C). Compared with that of the control group and ASO-NC-transfected cells, the expression of RhoE was significantly reduced in the ASO-miR-10-transfected cells. In addition, the expression of multi-drug resistance-associated genes MDR1 and MRP1 was investigated and the results showed that the mRNA and protein expression levels of MDR1 and MRP1 were significantly decreased following miR-10a silencing. Furthermore, as shown in Fig. 2D, there was an significant increase in the expression and activity of caspase-3/8 in miR-10a-silenced A549/DDP cells. These results indicated that miR-10a regulated the expression of apoptosis-associated genes in order to enhance drug resistance; therefore, Bcl-2, Survivin, RhoE and caspase-3/8 may be potential targets for the inhibition of apoptosis in A549/DDP cells.

miR-10a silencing suppresses the drug efflux of A549/DDP cells. The drug-resistance mechanisms of tumors were reported to be established due to the efflux function of cells $(10,11)$. It was therefore hypothesized that the effect of miR-10a silencing on DDP sensitivity may, at least in part, be due to the suppression of the efflux ability of cells. In the present study, the efflux ability of cistplatin-resistant A549/DDP cells on rhodamine-123 dye was evaluated following miR-10a silencing. As shown in Fig. 3A, the concentration of rhodamine-123 in A549/DDP cells following ASO-miR-10a transfection was significantly increased compared with that of the A549/DDP and ASO-NC groups, indicating that the efflux capacity was significantly reduced. Furthermore, the expression of P-gp on the cell surface of A549/DDP cells was examined using flow cytometry; as shown in Fig. 3B, miR-10a silencing markdely reduced the concentration of P-gp compared to that of the A549/DDP and ASO-NC groups. These experimental results indicated that drug efflux had an important role in increasing the drug sensitivity of A549/DDP cells following miR-10a silencing.

MiR-10a silencing regulates drug resistance associated signaling pathway. Tumor cells are able to enhance their proliferation, survival, invasion and metastasis abilities via the secretion of cytokines (12). In the present study, the secretion of TGF- $\beta$ was evaluated using an ELISA assay and the results demonstrated that miR-10a silencing decreased the secretion of TGF- $\beta$ (Fig. 4A).

The activation of drug resistance-associated signaling pathways was reported to be an important indicator of tumor drug resistance (13). In the present study, the phosphorylation levels of Sma and Madrelated protein (Smad)2, STAT3 and STAT5 in A549/DDP cells was determined following miR-10a silencing. As shown in Fig. 4B, the phosphorylation levels of Smad2, STAT3 and STAT5 were significantly reduced compared with those of the control group. Furthermore, the luciferase reporter gene method was used to detect the activity of the transcription factors HIF and eIF4E in A549/DDP cells. As shown in Fig. 4C, following miR-10a silencing, the activities of HIF and eIF4E were significantly reduced. These experimental results indicated that miR-10a regulated the TGF- $\beta /$ Smad2/STAT3/STAT5 signaling pathway and downstream transcription factors HIF and eIF4E in order to induce DDP resistance in A549 cells.

\section{Discussion}

miRNA has been the subject of studies worldwide and as a results of the continuous advancements of the associated 
A

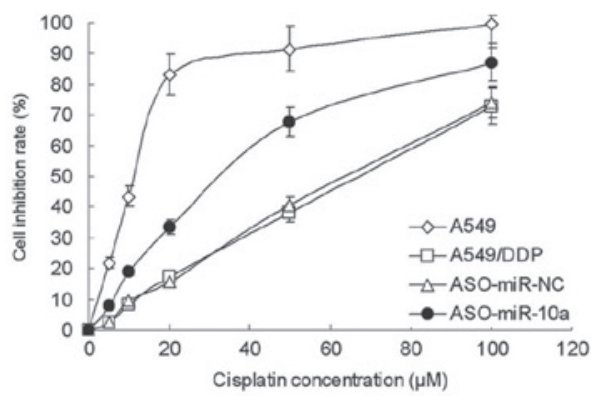

C

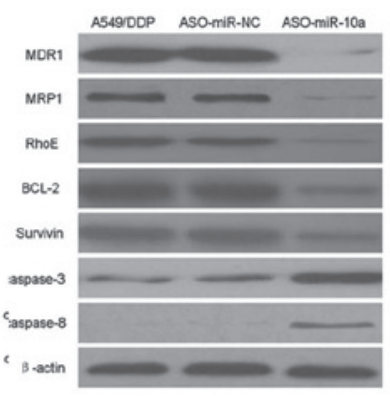

D
B

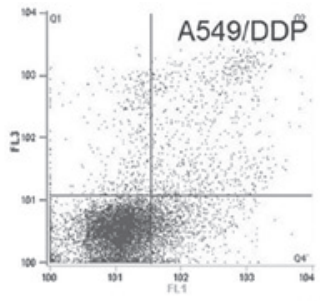

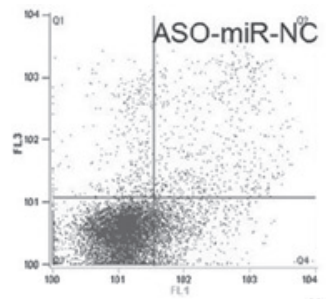
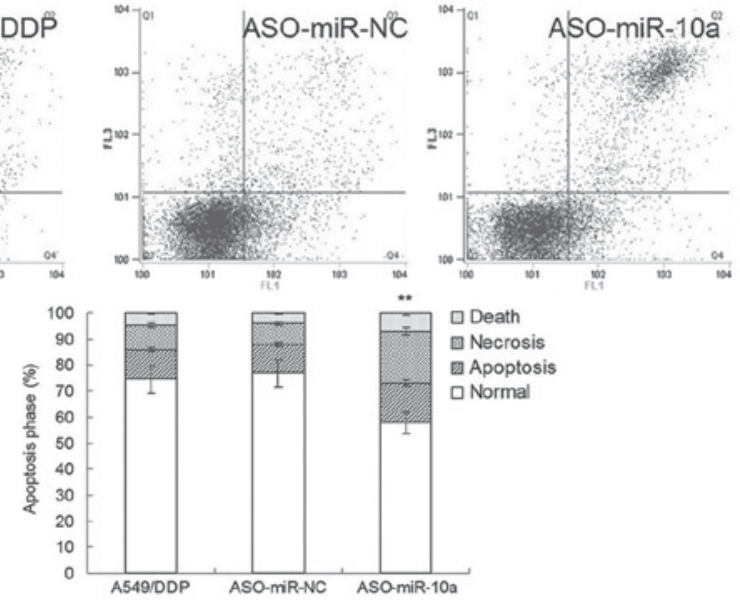
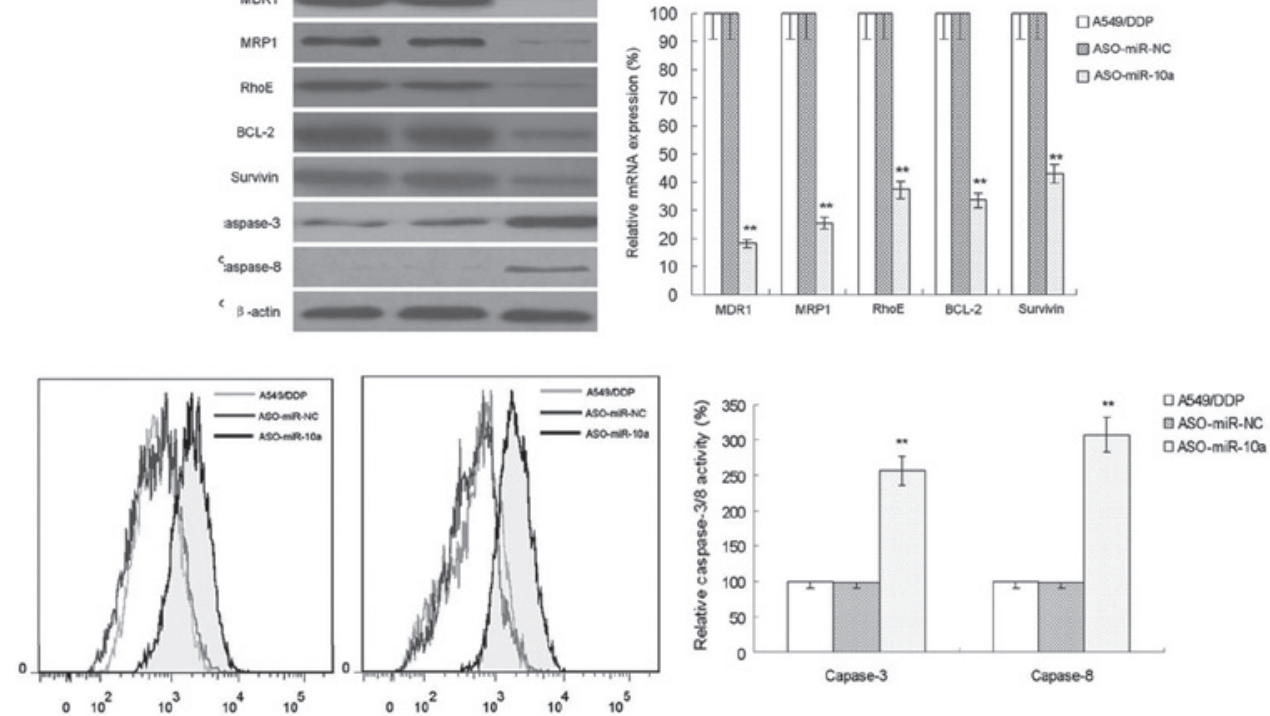

Figure 2. miR-10a silencing reverses DDP resistance in A549/DDP cells. (A) MTS assay for cell inhibition demonstrated the effect of miR-10a silencing on DDP sensitivity in cells in each group $(\mathrm{n}=10)$. (B) Flow cytometric analysis of the apoptotic rate of A549/DDP cells following transfection of ASO-miR-NC or the miR-10a inhibitor ASO-miR-10a $(\mathrm{n}=3)$. (C) Western blot analysis and quantitative polymerase chain reaction were used to detect the protein and mRNA expression, respectively, of MDR1, MRP1, RhoE, Bcl-2 and survivin in A549/DDP cells following transfection of ASO-miR-NC or ASO-miR-10a (n=5) (D) Flow cytometric analysis of caspase-3/8 activity in A549/DDP cells following transfection of ASO-miR-NC or ASO-miR-10a ( $\mathrm{n}=3$ ). Values are presented as the mean \pm standard deviation. ${ }^{* *} \mathrm{P}<0.05$ vs. the A549/DDP group. DDP, cisplatin; A549 group, control; A549/DDP group, DDP-resistant A549 cells without treatment; ASO-miR-NC group, negative control group of A549/DDP cells transfected with miR-10a; ASO-miR-10a group, A549/DDP cells transfected with an miR-10a inhibitor; miR, microRNA; MDR1, multidrug resistance protein 1; MRP1, multidrug resistance-associated protein 1; Bcl-2, B-cell lymphoma 2.

research, the important roles of miRNA in human physiological and pathological processes have been gradually elucidate; most notably, its roles in cancer (14).

DDP has been widely used in the clinical treatment of cancer; however, the occurrence of DDP resistance has hindered its application (15). The present study demonstrated that miR-10a had an important role in the DDP-resistant mechanisms of non-small cell lung cancer A549/DDP cells. The expression of miR-10a in DDP-resistant A549/DDP cells was found to be significantly increased compared to that of normal A549 cells. Following miR-10a silencing in A549/DDP cells, DDP sensitivity was significantly improved, suggesting that the increase of miR-10a is a key mechanism of DDP resistance in lung cancer. In addition, the results of the present study demonstrated that miR-10a silencing increased the apoptotic rate of A549/DDP cells; furthermore, the expression levels of Bcl-2 and Survivin were markedly reduced, indicating that miR-10a inhibited apoptosis via the inhibition of apoptosis-associated gene expression. RhoE is a member of the small guanine triphosphatase protein superfamily and previous studies have reported that RhoE had an important role in the drug resistance of tumors (16). The present study found that miR-10a regulated the expression of RhoE; following miR-10a silencing in A549/DDP cells, the expression of RhoE was significantly decreased, confirming the association between miR-10a and RhoE.

The most common mechanism of drug resistance in tumor cells is the decrease of intracellular drug concentration caused by increased drug efflux of associated proteins; MDR1 and MRP1 were reported to be the most important genes involved in enhancing drug efflux $(17,18)$. In the present study, inhibition of miR-10a was found to significantly reduce the expression of MDR1 and MRP1, indicating that miR-10a promoted their expression and therefore promoted DDP resistance through the enhancement of drug efflux. 
A
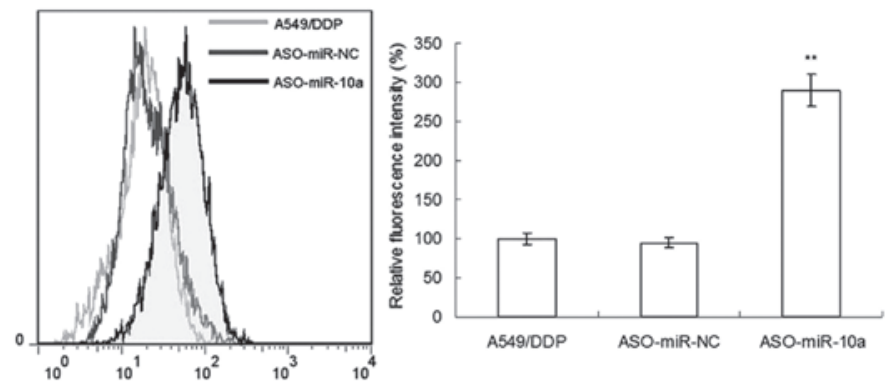

B
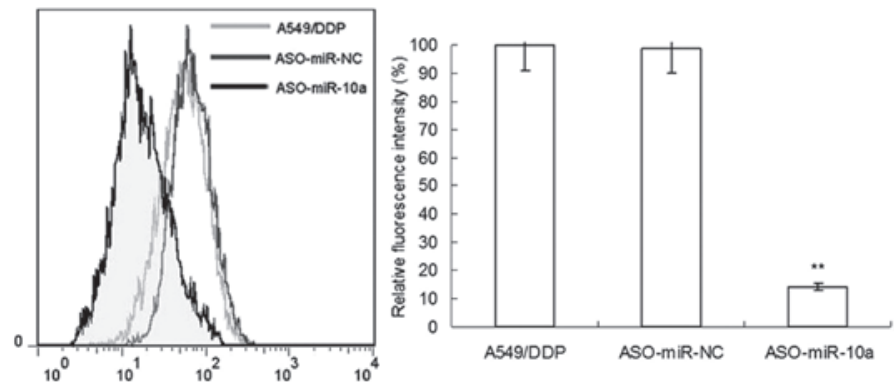

Figure 3. miR-10a silencing decreases drug efflux in A549/DDP cells. Flow cytometric analysis of (A) intracellular rhodamine-123 content and (B) P-gp expression in A549/DDP cells following transfection of ASO-miR-NC or the miR-10a inhibitor ASO-miR-10a. Values are presented as the mean \pm standard deviation ( $\mathrm{n}=3$ ). ${ }^{* *} \mathrm{P}<0.05$ vs. the A549/DDP group. DDP, cisplatin; A549/DDP group, DDP-resistant A549 cells without transfection; ASO-miR-NC group, negative control group of A549/DDP cells transfected with miR-10a; ASO-miR-10a group, A549/DDP cells transfected with an miR-10a inhibitor; miR, microRNA; P-gp, P-glycoprotein.

A

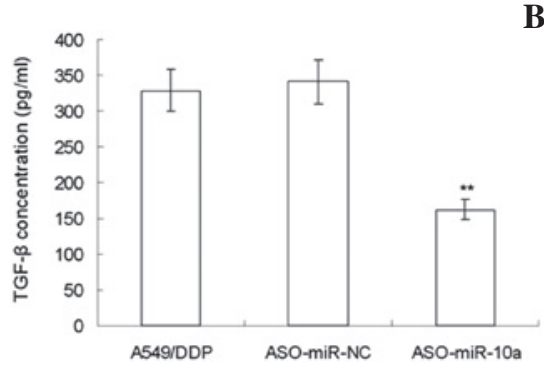

B

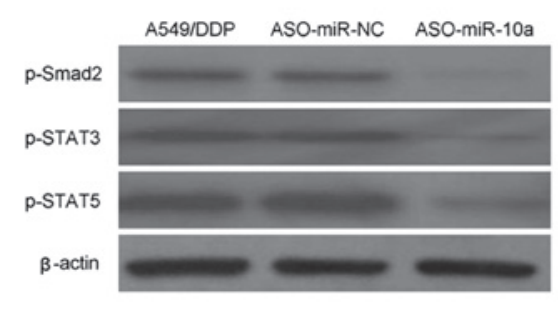

A549DDP

¿ASO-miR-NC

口ASO-miR-10a

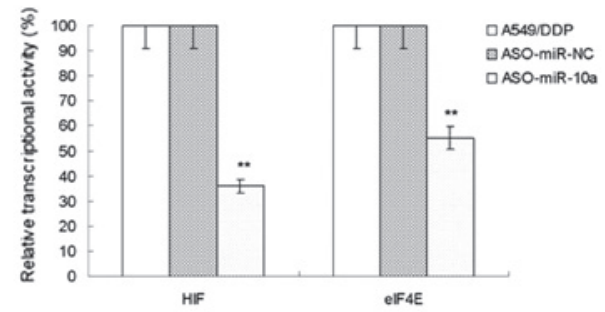

Figure 4. The effect of MiR-10a silencing on regulating the drug resistance-related signaling pathway of A549/DDP cell lines. (A) ELISA assays were used to evaluate the secretion of TGF- $\beta$ in A549/DDP cells following transfection of ASO-miR-NC or the miR-10a inhibitor ASO-miR-10a (n=10). (B) Western blot analysis of phosphorylated levels of Smad2, STAT3 and STAT5 in in A549/DDP cells following transfection of ASO-miR-NC or the miR-10a inhibitor ASO-miR-10a. (C) Luciferase gene report assay was used to detect the activity of HIF and eIF4E in A549/DDP cells following transfection of ASO-miR-NC or the miR-10a inhibitor ASO-miR-10a $(\mathrm{n}=10)$. Values are presented as the mean \pm standard deviation ${ }^{* *} \mathrm{P}<0.05$ vs. the A549/DDP group. DDP, cisplatin; miR, microRNA; TGF- $\beta$, transforming growth factor $\beta$; Smad, Sma- and Mad-related protein; STAT, signal transducer and activator of transcription; HIF, hypoxia-inducible factor; eIF4E, eukaryotic translation initiation factor 4E; p-, phosphorylated; A549/DDP group, DDP-resistant A549 cells without transfection; ASO-miR-NC group, negative control group of A549/DDP cells transfected with miR-10a; ASO-miR-10a group, A549/DDP cells transfected with an miR-10a inhibitor.

The secretion of cancer-promoting cytokines was reported to be another important mechanism for tumor cells to maintain proliferation and vitality through affecting the tumor microenvironment and signal pathways (19). TGF- $\beta$ has been shown to be an important cytokines involved in tumor promotion (20). The present study demonstrated that miR-10a silencing suppressed the expression of TGF- $\beta$ in tumor cells, suggesting that miR-10a may enhance drug resistance through affecting the expression of TGF- $\beta$.

Tumor cells may maintain their survival abilities through activating certain signaling pathways. The present study demonstrated that miR-10a was able to regulate the TGF- $\beta /$ Smad2/STAT3/STAT5 signaling pathway, which has been reported to be one of the most important drug 
resistance-associated signaling pathways involved in drug efflux and inhibiting apoptosis (21). The results of the present study showed that miR-10a promoted the activity of Smad2, STAT3 and STAT5 as well as the downstream transcriptional factors of HIF and eIF4E in order to induce DDP resistance in A549 cells.

In conclusion, the present study demonstrated that miR-10a had an important role in promoting drug resistance in tumors through enhancing drug efflux and inhibiting apoptosis via upregulation of MDR1, MRP1 and RhoE expression. In addition, miR-10a promoted the expression of TGF- $\beta$ as wells as regulated the activity of the Smad2/STAT3/STAT5 pathway and its downstream transcriptional factors of HIF and IF4E, which may be the potential mechanism of drug resistance in A549 cells. Therefore, miR-10a may be an important drug target for improving cancer treatment; however, further studies are required to explore the clinical applications of miR-10a inhibitors.

\section{References}

1. Joshi P, Middleton J, Jeon YJ and Garofalo M: MicroRNAs in lung cancer. World J Methodol2: 5972, 2014.

2. Liu R, Liu X, Zheng Y, Gu J, Xiong S, Jiang P, Jiang X, Huang E, Yang Y, Ge D and Chu Y: MicroRNA7 sensitizes nonsmall cell lung cancer cells to paclitaxel. Oncol Lett 5: 21932200, 2014.

3. Cai J, Fang L, Huang Y, Li R, Yuan J, Yang Y, Zhu X, Chen B, Wu J and Li M: miR-205 targets PTEN and PHLPP2 to augment AKT signaling and drive malignant phenotypes in non-small cell lung cancer. Cancer Res 73: 5402-5415, 2013.

4. Ayaz L, Görür A, Yaroğlu HY, Ozcan C and Tamer L: Differential expression of microRNAs in plasma of patients with laryngeal squamous cell carcinoma: potential early-detection markers for laryngeal squamous cell carcinoma. J Cancer Res Clin Oncol 139: 1499-1506, 2013.

5. Köhler CU, Bryk O, Meier S, Lang K, Rozynek P, Brüning T and Käfferlein HU: Analyses in human urothelial cells identify methylation of miR-152, miR-200b and miR-10a genes as candidate bladder cancer biomarkers. Biochem Biophys Res Commun 438: 48-53, 2013.

6. Zhu Y, Liu XJ, Yang P, Zhao M, Lv LX, Zhang GD, Wang Q and Zhang L: Alkylglyceronephosphate synthase (AGPS) alters lipid signaling pathways and supports chemotherapy resistance of glioma and hepatic carcinoma cell lines. Asian Pac J Cancer Prev 7: 32193226, 2014.

7. Ulasli SS, Celik S, Gunay E, Ozdemir M, Hazman O, Ozyurek A, Koyuncu T and Unlu M: Anticancer Effects of Thymoquinone, Caffeic Acid Phenethyl Ester and Resveratrol on A549 Non-small Cell Lung Cancer Cells Exposed to Benzo(a)pyrene. Asian Pac J Cancer Prev 14: 6159-6164, 2013.

8. Sun PL, Jin Y, Kim H, Seo AN, Jheon S, Lee CT and Chung JH: Survivin expression is an independent poor prognostic marker in lung adenocarcinoma but not in squamous cell carcinoma. Virchows Arch 463: 427-436, 2013.
9. Zhang C, Zhou F, Li N, Shi S, Feng X, Chen Z, Hang J, Qiu B, Li B, Chang S, et al: Overexpression of RhoE has a prognostic value in non-small cell lung cancer. Ann Surg Oncol 14: 2628-2635, 2007

10. Prochazka L, Koudelka S, Dong LF, Stursa J, Goodwin J, Neca J, Slavik J, Ciganek M, Masek J, Kluckova K, et al: Mitochondrial targeting overcomes ABCA1-dependent resistance of lung carcinoma to $\alpha$-tocopheryl succinate. Apoptosis 18: 286-299, 2013.

11. Minami T, Kijima T, Otani Y, Kohmo S, Takahashi R, Nagatomo I, Hirata H, Suzuki M, Inoue K, Takeda Y, et al: HER2 as therapeutic target for overcoming ATP-binding cassette transporter-mediated chemoresistance in small cell lung cancer. Mol Cancer Ther 11: 830-841, 2012.

12. Wang YS, Miao LY, Liu L, Cai HR, Ding JJ, Ren SX, Zhou CC and Schmid-Bindert G: Serum cytokine levels in patients with advanced non-small cell lung cancer: correlation with clinical outcome of erlotinib treatment. Chin Med J (Engl) 126: 3931-3935, 2013.

13. Cha Y, Kim DK, Hyun J, Kim SJ and Park KS: A3 binds to TGF-beta receptor I and induces Smad-independent, JNK-dependent apoptosis in ovarian cancer cells. Cell Signal 25: 1245-1251, 2013.

14. Zhang K, Zhang Y, Liu C, Xiong Y and Zhang J: MicroRNAs in the diagnosis and prognosis of breast cancer and their therapeutic potential. Int J Oncol 3: 950958, 2014.

15. Li Y,Li L, Guan Y, Liu X, Meng Q and Guo Q: MiR-92b regulates the cell growth, cisplatin chemosensitivity of A549 non small cell lung cancer cell line and target PTEN. Biochem Biophys Res Commun 440: 604-610, 2013.

16. Avasarala S, Bikkavilli RK, Van Scoyk M, Zhang W, Lapite A, Hostetter L, Byers JT, Heasley LE, Sohn JW and Winn RA: Heterotrimeric g-protein, g $\alpha 16$, is a critical downstream effector of non-canonical wnt signaling and a potent inhibitor of transformed cell growth in non small cell lung cancer. PLoS One 8: e76895, 2013.

17. Surowiak P,Pawełczyk K, Maciejczyk A, Pudełko M, Kołodziej J, Zabel M, Murawa D, Drag M, Gansukh T, Dietel M and Lage H: Positive correlation between cyclooxygenase 2 and the expression of ABC transporters in non-small cell lung cancer. Anticancer Res 28 (5B): 2967-2974, 2008.

18. Ak Y, Demirel G and Gülbas Z: MDR1, MRP1 and LRP expression in patients with untreated acute leukaemia: correlation with $99 \mathrm{mTc}-\mathrm{MIBI}$ bone marrow scintigraphy. Nucl Med Commun 28: 541-546, 2007.

19. Wang L, Deng Q, Wang J, Bai X, Xiao X, Lv HR, Zhao MF and Liu PJ: Effect of CIK on multidrugresistance reversal and increasing the sensitivity of ADR in K562/ADR cells. Oncol Lett 4: 17781782, 2014.

20. Kin R, Kato S, Kaneto N, Sakurai H, Hayakawa Y,Li F, Tanaka K, Saiki I and Yokoyama S: Procyanidin C1 from Cinnamomi Cortex inhibits TGF- $\beta$-induced epithelial-to-mesenchymal transition in the A549 lung cancer cell line. Int J Oncol 43:1901-1906, 2013.

21. Tu B, Peng ZX, Fan QM, Du L, Yan W and Tang TT: Osteosarcoma cells promote the production of pro-tumor cytokines in mesenchymal stem cells by inhibiting their osteogenic differentiation through the TGF- $\beta / \operatorname{Smad} 2 / 3$ pathway. Exp Cell Res 320:164-173, 2014. 